This item is the archived peer-reviewed author-version of:

\title{
Motion sickness and sopite syndrome associated with parabolic flights : a case report
}

\section{Reference:}

Van Ombergen Angelique, Lawson Ben D., Wuyts Floris.- Motion sickness and sopite syndrome associated with parabolic flights : a case report

The international journal of audiology / Royal National Institute for the Deaf; British Society of Audiology - ISSN 1708-8186 (2015), p. 1-6

Full text (Publishers DOI): http://dx.doi.org/doi:10.3109/14992027.2015.1111526 
Complete manuscript title: Motion sickness and sopite syndrome associated with parabolic flights: a case report.

Manuscript ID: TIJA-2015-08-0219

Running head: motion sickness and sopite in parabolic flight.

Authors: Angelique Van Ombergen ${ }^{1}$, Ben D. Lawson ${ }^{2}$, Floris L. Wuyts ${ }^{1}$

Affiliations:

${ }^{1}$ Antwerp University Research Centre for Equilibrium and Aerospace (AUREA), University of Antwerp, Groenenborgerlaan 171, 2020 Antwerp. Belgium.

${ }^{2}$ U.S. Army Aeromedical Research Laboratory, Fort Rucker, Alabama, U.S.A. 36362

Corresponding Author: Angelique Van Ombergen, MSc, Antwerp University Research centre for Equilibrium and Aerospace (AUREA), University of Antwerp, Groenenborgerlaan 171, 2020 Antwerpen, Belgium

Tel: +32 (0)3 8213307

Email: angelique.vanombergen@uantwerp.be

Key Words: motion sickness, sopite syndrome, parabolic flight, vestibular system.

\section{Acronyms \& abbreviations:}

Diffusion Tensor Imaging: DTI; Epworth Sleepiness Scale: ESS; European Space Agency: ESA; Excessive daytime sleepiness: EDS; Free-floating: FF; Magnetic Resonance Imaging: MRI; Motion Sickness Assessment Questionnaire: MSAQ; Parabolic flight: PF; Positive and Negative Affect Scale: PANAS; Prediction Interval: PI; Standard deviation: SD. 


\begin{abstract}
Objective: The purpose of this paper was to provide an extensive description of a case of sopite syndrome, a manifestation of motion sickness scarcely described in the literature.

Design: The following questionnaires were used to assess sopite syndrome symptoms pre, during and post parabolic flight; Misery Scale rate, Positive and Negative Affect Scale, the Motion Sickness Assessment Questionnaire and the Epworth Sleepiness Scale.

Study sample: Case report of a 35-year old female compared to a control group $(n=26)$.

Results: We describe of sopite syndrome during parabolic flight. The subject reported drowsiness and she noted mood changes such as irritation and annoyance. Furthermore, she was not able to perform her assigned task. Her symptoms escalated into extreme fatigue and the inability to stay awake. Nauseogenic symptoms improved, but soporific symptoms persisted for several hours after the motion stimulus (i.e. parabolic flight).

Conclusions: This case points towards the need for controlled studies to assess the prevalence of this syndrome among the general population and select groups. Future research should focus on developing tests for personnel screening and explore treatment options.
\end{abstract}




\section{Introduction}

Motion sickness is a common, though complex syndrome in otherwise healthy people exposed to real or apparent motion challenges. Its primary signs and symptoms are nausea, vomiting, increased salvation, pallor and cold sweating, yet associated signs and symptoms can extend to headache, dizziness, drowsiness, yawning, sighing, flatulence and loss of body weight (Miller \& Graybiel 1970; Schmäl 2013). Anyone with a normal functioning vestibular system is at risk for motion sickness. However, research has suggested that age, personality traits and the specific motion environment are an important factor in one's susceptibility to motion sickness (Lackner 2014; Golding 2006). Provocative stimuli can be real (physical) or apparent (i.e. visual or virtual) motion(Kennedy et al. 2010; Golding 2006).

Although the existence of motion sickness as a syndrome has been long acknowledged, it was not until 1976 that Gaybriel and Knepton identified the sopite syndrome (Graybiel \& Knepton 1976), a facet of motion sickness that often goes unrecognized or missed (Lawson \& Mead 1998). Previous papers stated that in some cases, depending on the individual and the environmental stimulus, sopite syndrome can even present as the sole or main clinical manifestation of motion sickness (Graybiel \& Knepton 1976; Lawson \& Mead 1998). Sopite syndrome is an entity that develops as a result of exposure to real or apparent motion, just as is the case with motion sickness. Main symptoms are drowsiness, mood changes, irritability, lethargy and the inability to concentrate (Graybiel \& Knepton 1976; Lawson \& Mead 1998). Other associated symptoms extend to apathy, yawning, disinclination to work (either physical or mental), desire to be left alone, disinterest in group activities, sleep disturbances, melancholy and depression(Lackner 2014; Graybiel \& Knepton 1976; Lawson \& Mead 1998). The time course of sopite syndrome is dependent on the specific situation, but it has been reported that symptoms 
can appear quickly, even after a short or weak stimulus, and symptoms can persist even after cessation of the motion stimulus (Lawson \& Mead 1998). Unfortunately, sopite syndrome is often missed due to its non-specific symptoms or because other factors such as sleep deprivation and other forms of fatigue related to operational settings confounding it (Lawson \& Mead 1998; Matsangas \& McCauley 2014a). The fact that symptoms are sometimes mild (e.g. compared to clearly observable motion sickness signs such as emesis) could also lead to unrecognized sopite, while other clinical problems such as depression may lead to misdiagnosis (Lawson \& Mead 1998).

Motion sickness and sopite syndrome have been studied and described thoroughly in multiple environments, including land travel (Golding 2006), sea travel (Lawson \& Mead 1998; Matsangas et al. 2010), air travel (Graybiel \& Knepton 1976; Lawson \& Mead 1998), optokinetic drums (Kiniorski et al. 2004), virtual environments (Lawson \& Mead 1998; Kennedy et al. 1998; Hettinger et al. 1990; Golding 2006), rotating rooms (Graybiel \& Knepton 1976; Lawson \& Mead 1998) and spaceflight (Lackner \& DiZio 2006; Lackner 2014). In addition, motion sickness has been studied in animals and humans during parabolic flights (Suri et al. 1979; Schlegel et al. 2001; Harm \& Schlegel 2002; Hilbig et al. 2002; Strewe et al. 2012). Although the occurrence of sopite syndrome during parabolic flights is widely known about and expected, thorough assessment and description of this particular motion sickness facet remains limited and anecdotal (Lawson \& Mead 1998).

Motion sickness and sopite syndrome may have a serious impact operationally because they occur commonly (Matsangas \& McCauley 2014a) and it is suggested that they could insidiously lead to work inefficiency and an unsafe work environment (Matsangas \& McCauley 2014a). Furthermore, a recent study showed that mild motion sickness and sopite syndrome have 
a negative impact on cognitive performance and the cognitive effort focused on performing a task (Matsangas et al. 2014). Identification of motion sickness and specifically sopite syndrome is therefore crucial.

We here describe a particular case of motion sickness and sopite syndrome in parabolic flight. To the best of our knowledge, this is the first extensive report on this matter. By providing this report, we hope to give an insight into an underrecognized and underreported condition and to stimulate future (prospective) research into this complicated phenomenon.

\section{Methods}

All data was collected as part of the ESA BRAIN Diffusion Tensor Imaging (BRAIN DTI) project, an ongoing investigation to study neuroplasticity due to parabolic flight as well as spaceflight induced microgravity and specific vestibular lesions (Clément \& Ngo-Anh 2013; Van Ombergen et al. 2013; Demertzi et al. 2015).

\subsection{Subjects}

In total, 27 participants were included (18 males, mean age (SD): 32.3 (7.4) years). Data was collected over 4 parabolic flight campaigns, scheduled between April 2014 and June 2015. All participants had to pass a Class 3 Aviation medical exam and underwent a MRI scan (as part of the BRAIN DTI project) prior to the flight. Participants had to be first-time flyers to be included and exclusion criteria were: 1) medical conditions in the acute phase e.g. orthopaedic injury, 2) vestibular, psychiatric and/or neurological diseases and 3) smokers. 
This study was approved by the local ethics committee (IRB number 13/38/357) and the ESA medical board. Each participant provided a signed informed consent form. All investigations have been conducted according to the principles expressed in the Declaration of Helsinki.

\subsection{Medication and parabolic flight}

Prior to the parabolic flight, all participants received a subcutaneous injection of scopolamine $(0.25 \mathrm{mg} / \mathrm{ml})$, an anti-emetic drug. This was administered approximately 2 hours before the first parabola by the campaign medical doctor. During the flight (onboard the Airbus A-300 Zero-G and the Airbus A-310 Zero-G), 31 parabolic maneuvers were conducted to evoke zero G. Each parabola started with a pull-up phase and ended with a pull-out phase at $1.8 \mathrm{~g}$, both lasting about $20 \mathrm{sec}$. The duration of the zero $\mathrm{G}$ condition was about $21 \mathrm{sec}$. The time between each parabola start is approximately 3 minutes. The time between the end of a parabola and the start of the next one is around $1 \mathrm{~min}$ and 45 seconds. After every set of 5 parabolas, a 5 -minute break is implemented. However, between parabola 15 and parabola 16, a longer break of 8 minutes is employed. During the first five parabolas, the subject was seated to enhance adaptation to the peculiar sensation of weightlessness and hypergravity and to limit the initial activity in the novel environment. The latter is suggested to be an effective way of decreasing motion sickness (Lackner 2014). After these five parabolas, the subject had to float in the restricted free-floating zone, as part of the protocol of the BRAIN DTI experiment.

\subsection{Questionnaire assessment}

\subsubsection{Motion Sickness Assessment Questionnaire (MSAQ)}

The MSAQ is a questionnaire comprising four subscales, all assessing a different aspect of 
motion sickness (Gastrointestinal, Central, Peripheral and Sopite-related) (Gianaros \& Muth 2001). The score on each subscale resembles how severe the symptoms are for each of the different categories and the total motion sickness score can be calculated by adding all subscale scores. All MSAQ scores range from approximately $11.11 \%$ (minimum) to $100 \%$. Participants had to complete the MSAQ immediately after the parabolic flight.

\subsubsection{Epworth Sleepiness Scale (ESS)}

The ESS is a standardized and validated questionnaire assessing the likelihood that the participant will fall asleep during certain activities (Johns 1991). The ESS consists of 8 questions that describe daily situations that can induce sleepiness. Each question is graded from score 0 ("not likely to fall asleep") to score 3 ("very likely to fall asleep"). The ESS score ranges from 0 (minimum) to 24 (maximum). A score of 10 is used as a cut-off value to make a distinction between normal individuals (ESS $\leq 10)$ and individuals suffering from excessive daytime sleepiness (ESS $>10)$. The ESS was incorporated to assess the typical fatigue and drowsiness associated with sopite syndrome and was filled in twice by each participant: prior to and immediately after the parabolic flight.

\subsubsection{Positive and Negative Affect Scale (PANAS)}

The PANAS is a valid 20-item self-report measure of positive and negative affect (Watson et al. 1988). Ten positive affect items were rated on a 5-point Likert scale from 1 (very slightly or not at all) to 5 (extremely) regarding the present moment (at that time) with possible total scores ranging from 10 (minimum) to 50 (maximum). In addition, ten negative affect items were rated, also on a 5-point Likert scale with the same properties as for the positive statements. Momentary mean scores are $29.7(\mathrm{SD}=7.9)$ and $14.8(\mathrm{SD}=5.4)$ for the Positive Affect Score and Negative 
Affect Score, respectively. The PANAS was completed prior to and immediately after the parabolic flight.

\subsection{Sopite case report}

A 35-year old female journalist participated in the BRAIN DTI project and in parallel, she was supposed to observe and conduct brief interviews with experimenting teams on board during breaks between parabolic maneuvers. She had no history of neuro-vestibular pathology and had a normal preflight MRI scan. Quite soon during the parabolic flight, she reported that she started experiencing general malaise, nausea, cold sweat and drowsiness. After a while, she started feeling irritated, annoyed and there was an inability to perform the task she was assigned to do. By the end of the parabolic flight, these symptoms escalated into extreme fatigue, the inability to stay awake and being close to fainting. The symptoms persisted for a couple of hours after landing. After the flight, she also reported being easily upset and the inability to control herself. During a phone conversation with one of the investigators, approximately 3 hours after the flight, she was very rude and impolite without any reason. The subject described it as: "normally I think before I speak, but I was not able to do so then and I had no self control". She apologized for this behavior the day after. This was in contrast to the investigator's experience with her beforehand, as she was always very friendly, professional and dedicated. She wasn't able to finish the interviews she was supposed to do during the flight. She reported having a normal night of sleep before commencing the parabolic flight. Her emotional state, as described above, was assessed through verbal reports. Her results on the questionnaires, in comparison to the control group, are shown and discussed below. 


\subsection{Statistical analyses}

Statistical analysis was performed with SPSS version 21 (IBM Corp., Version 21.0. Armonk, NY: IBM Corp). All data was checked for normality with Kolmogorov-Smirnov tests. To investigate the difference between the sopite case subject and the control group, we calculated the $95 \%$ prediction interval $(\mathrm{PI}=[$ mean $\pm 2 \mathrm{SD}])$ for each of the questionnaire assessments. The values at which the score from the sopite case did not fall within the 95\% PI, were considered as significantly different from the control group.

\section{Results}

\subsection{Sopite case report}

The subject filled in the following questionnaires pre- and post parabolic flight: the Positive and Negative Affect Scale (PANAS) (Watson et al. 1988) and the Epworth Sleepiness Scale (ESS) (Johns 1991). The Motion Sickness Assessment Questionnaire (MSAQ) (Gianaros \& Muth 2001) was assessed only after the parabolic flight (Tab. 3). The pre-flight score of our subject on the PANAS is comparable to the mean scores for momentary assessment (mean score $=29.7(\mathrm{SD}=7)$ of the positive affect, mean score $=14.8(\mathrm{SD}=5.4))($ Watson et al. 1988). However, post-flight scores show a reduction for positive affect and increased negative affect when compared with the mean scores (Watson et al. 1988) and preflight scores. Preflight ESS score was relatively high $(=10)$ to start with, but the post-flight ESS score increased severely and was extremely high $(=23)$. Furthermore, MSAQ was assessed after the parabolic flight. The overall score is high and our subject scores relatively high on all subscales. Moreover, the subject scored $100 \%$ on the sopiterelated subscale, indicating these symptoms were the most present and the most severe. 


\subsection{Sopite case report versus group}

More interestingly, we also compared the subject's scores with the $95 \%$ prediction intervals of the questionnaire scores of the control group $(\mathrm{n}=26)$. There was no significant difference in age between the sopite subject and the rest of the participants.

\subsubsection{Pre flight}

Preflight, all scores of the sopite case fell within the 95\% PI of the control group. In other words, we could not find any differences preflight for the ESS, the PANAS positive score and the PANAS negative score.

\subsubsection{Post flight}

When we compared the results on the questionnaires filled in after the flight, the values from all questionnaires (PANAS positive, PANAS negative, ESS, MSAQ) fell outside of the 95\% PI of the control group. A summary can be found in Table 1.

- insert Table 1 about here -

\section{Discussion}

\subsection{Sopite case report}

The case report we are describing fulfills the recently revised definition of the sopite syndrome by Matsangas et al (Matsangas \& McCauley 2014a). In addition, our case is also in line with earlier descriptions of sopite syndrome: a healthy individual (Lackner 2014), multiple symptoms (Graybiel \& Knepton 1976; Lawson \& Mead 1998; Matsangas \& McCauley 2014a), 
inability to perform/focus on a designated task (Matsangas \& McCauley 2014a; Matsangas et al. 2014) and the fact that typical sopite syndrome symptoms persist, even after the motion stimulus, is also in line with earlier findings (Lawson \& Mead 1998).

The subject did not have a known history of sleep abnormalities, or any abnormalities in vestibular, neurological or mental function. Also, we did not find any differences between the sopite subject and the control group for any of the preflight questionnaires. These findings lead us to infer that the soporific symptoms we observed are probably not solely attributable to an endogenous abnormality that emerged on its own or that was triggered by non-motion aspects of the flight such as anxiety and/or excitement.

An important factor that should be kept in mind is the scopolamine injection (i.e. subcutaneous injection, $0.25 \mathrm{mg} / \mathrm{ml})$. Research has shown that the side effects of anti-emetic medication, such as scopolamine, may exacerbate existing drowsiness (Lackner 2014; Graybiel \& Knepton 1976; Lawson \& Mead 1998). In line with this, it is important to realize that anti-emetic medication is administered to improve performance in spaceflight and aviation crew. However, due to sopite syndrome, medication might not adequately improve performance (Lackner 2014; Graybiel \& Knepton 1976; Lawson \& Mead 1998; Matsangas \& McCauley 2014a). Correct identification of sopite effects in an individual is therefore crucial to minimize the negative impact on performance. However, one should be careful when interpreting this, since it is possible that the observed behavior in our subject is partially caused by the scopolamine. We explored this possibility indirectly by comparing the sopite subject to a control group who also received scopolamine and were all first time flyers as well. In addition, it has been shown that several biological variables can influence drug sensitivity, with age being one of the main factors. Since we found no difference in age between the sopite subject and the control group, the 
influence of age can be minimized (McMaster \& Carney 1985). Furthermore, it has been shown that scopolamine administration only leads to limited drowsiness, sedation and cognitive impairment in non-moving baseline conditions. This suggests a case of extreme sensitivity to sopite syndrome (i.e. due to motion) rather than purely extreme drug sensitivity (Wood et al. 1984; Simmons et al. 2010; Weerts et al. 2015). Additionally, transdermal scopolamine in moving situations showed to have minimal side effects such as drowsiness (Price et al. 1981). In any case, future research should focus on individual sensibility to scopolamine and sopite syndrome separately, as this may have important implications for personnel selection and motion sickness therapeutic strategies.

Up until several hours after the parabolic flight, when nauseogenic symptoms already disappeared, the subject still experienced a sense of 'depersonalization' of which the rude, impolite and borderline-aggressive behavior is suggestive. During the phone call, she spoke to one of the investigators of our experiment and this person realized that this behavior was highly likely part of sopite syndrome. If this were not the case, the situation could have escalated. Therefore, it should be kept in mind these mood changes can linger on for hours. The subject reported a daytime sleepiness with an ESS score of 10 pre-flight, which is high to begin with, however this was not significantly different from the scores of the control group. Post-flight however, the subject's ESS score was 23, which is much higher than the cut-off of 10 for excessive daytime sleepiness (EDS) (=ESS $\geq 10)$ (Johns 1991). This corroborates with previous studies who could not find a relation between daytime sleepiness and sopite syndrome (Lackner 2014).

The overall score on the MSAQ was high, showing a combination of both soporific and motion sickness symptoms. We suggest including a combination of more fitted questionnaires, 
such as the above-described ESS, PANAS and MSAQ scales for future studies. Ideally, all participants indulging in future parabolic flights should complete these questionnaires before and after. Not only would this help to get more insight in a specific case presenting with sopite as a result of parabolic flight, analysis of these results could also help to get a more extensive knowledge on sopite syndrome.

In addition, it was shown by a recent investigation that higher MSAQ scores (i.e. increased severity of motion sickness and soporific symptoms) are related to a reduced multitasking cognitive performance(Matsangas \& McCauley 2014b). This implies that the considerable increment of MSAQ scores on all subscales for the sopite subject is associated with a parallel decrement in cognitive performance. While this was only described for mild cases of motion sickness and sopite syndrome, our report suggests this to be also true for more extreme cases. Furthermore, this can lead to poor decision-making among personnel, crew and executives.

A distinction between "pure sopite" syndrome (i.e. soporific symptoms without any gastro-enteric symptoms) and sopite with some degree of gastro-enteric symptoms should be made. While the latter probably occurs often, "pure sopite" syndrome is assumed to be rare. Although our case fell outside of the $95 \%$ prediction interval values for the gastro-enteric and peripheral scale, the "degree" of this deviation is considerably smaller than for the sopite-related and the central subscale. This suggests that the abnormalities are highest for the soporific and central symptoms and therefore implies that our subject is situated more closely to "pure sopite" rather than a mixed gastro-enteric/soporific form, if the two are seen as a spectrum. Future studies should focus on this spectrum and more specifically on the exact incidence of "pure sopite" in a prospective manner. 


\subsection{Limitations and future studies}

The most prominent limitation is the fact that we are describing a single case report that had taken scopolamine medication. However, by comparing the results to an age-matched control group of 26 subjects (all first time flyers, all under scopolamine medication), we believe we have been able to make some credible inferences concerning this case report. Another very important limitation is the possibility that we are seeing extreme individual drug sensitivity rather than an extreme sensitivity to sopite syndrome. However, due to several reasons mentioned above, our report suggests to be a case of extreme sensitivity to sopite syndrome, rather than a purely sensitivity to scopolamine. Nevertheless, future research should focus on developing tests that allow for personnel screening since the consequences of sopite on task performance, efficiency and decision-making cannot be ignored and hold a potentially safety hazard. In addition, prospective studies should assess therapeutic possibilities since evidence on effective therapy and pharmaceutical interaction with anti-emetic drugs is scarce.

\subsection{Conclusion}

To the best of our knowledge, this is the first extensive case report on sopite syndrome during parabolic flight. This case points towards the need for controlled studies to assess the prevalence of this specific motion sickness syndrome, not only in a parabolic flight crew but also among the general population and among select groups such as naval personnel, aviation crew, astronauts and even people who are being exposed to virtual reality, simulators or 3D movies.

\section{Acknowledgements}

The authors would like to thank all participants for their cooperation and the following coordinating persons: Vladimir Pletser (European Space Agency), Alexandra Jaquement (Novespace, Bordeaux, France) \& Frédéric Gai 
(Novespace, Bordeaux, France). This work was supported by the European Space Agency (ISLRA 2009-1062 and

LS60-3), Belgian Science Policy (Belspo, Prodex) and the Research Foundation Flanders (Belgium - FWO

Vlaanderen, grant 11U6414N and 11U6416N). Angelique Van Ombergen is a research fellow for FWO Vlaanderen.

\section{References}

Clément, G. \& Ngo-Anh, J.T., 2013. Space physiology II: Adaptation of the central nervous system to space flightpast, current, and future studies. Eur. J. Appl. Physiol., 113, pp.1655-1672.

Demertzi, A. et al., 2015. Cortical reorganization in an astronaut's brain after long-duration spaceflight (in press). Brain Struct. Funct.

Gianaros, P. \& Muth, E., 2001. A questionnaire for the assessment of the multiple dimensions of motion sickness. Aviat. Sp. Environ. Med., 72, pp.115-119.

Golding, J.F., 2006. Motion sickness susceptibility. Auton. Neurosci. Basic Clin., 129(1-2), pp.67-76.

Graybiel, A. \& Knepton, J., 1976. Sopite syndrome: a sometimes sole manifestation of motion sickness. Aviat. Sp. Environ. Med., 47, pp.873-882.

Harm, D.L. \& Schlegel, T.T., 2002. Predicting motion sickness during parabolic flight. Auton. Neurosci. Basic Clin., 97, pp.116-121.

Hettinger, L. et al., 1990. Vection and simulator sickness. Mil Psychol, 2(3), pp.171-81.

Hilbig, R. et al., 2002. Susceptibility to motion sickness in fish: a parabolic aircraft flight study. J Gravit Physiol, 9(1), pp.29-30.

Johns, M.W., 1991. A new method for measuring daytime sleepiness: the Epworth sleepiness scale. Sleep, 14, pp.540-545.

Kennedy, R., Stanney, K. \& Lawson, B., 1998. Independence of different cybersickness aftereffects: basis for a theory. Aviat Sp. Env. Med, 43, pp.181-92.

Kennedy, R.S., Drexler, J. \& Kennedy, R.C., 2010. Research in visually induced motion sickness. Appl. Ergon., 41(4), pp.494-503.

Kiniorski, E.T. et al., 2004. Sopite symptoms in the optokinetic drum. Aviat. Sp. Environ. Med., 75, pp.872-875.

Lackner, J.R., 2014. Motion sickness: More than nausea and vomiting. Exp. Brain Res., 232, pp.2493-2510.

Lackner, J.R. \& DiZio, P., 2006. Space motion sickness. Exp. Brain Res., 175, pp.377-399.

Lawson, B.D. \& Mead, A.M., 1998. The sopite syndrome revisited: Drowsiness and mood changes during real or apparent motion. Acta Astronaut., 43, pp.181-192. 
Matsangas, P. et al., 2010. Personnel physical activity levels on naval vessels - evidence for soporific and fatigue effects? In Proceedings of the International Conference of Human Performance at Sea (HPAS).

Matsangas, P. \& McCauley, M., 2014a. Sopite syndrome: a revised definition. Aviat Sp. Env. Med, 85, pp.672-3.

Matsangas, P. \& McCauley, M., 2014b. Yawning as a behavioural marker of mild motion sickness and sopite syndrome. Aviat Sp. Env. Med, 85, pp.658-661.

Matsangas, P., McCauley, M. \& Becker, W., 2014. The effect of mild motion sickness and sopite syndrome on multitasking cognitive performance. Hum. Factors, 75, pp.872-5.

McMaster, S.B. \& Carney, J.M., 1985. Changes in drug sensitivity following acute and chronic exercise. Pharmacol Biochem Behav, 23, pp.191-194.

Miller, E.F. \& Graybiel, A., 1970. A provocative test for grading susceptibility to motion sickness yielding a single numerical score. Acta Otolaryngol. Suppl., 274, pp.1-20.

Van Ombergen, A. et al., 2013. Neuroplasticity in astronauts and patients with vestibular lesions studied with novel MRI methods: proof of concept. In 19th IAA Humans in Space: linking the challanges of space exploration with medicine on Earth. Cologne, Germany.

Price, N. et al., 1981. Transdermal scopolamine in the prevention of motion sickness at sea. Clin Pharmacol Ther, 29(3), pp.414-419.

Schlegel, T.T. et al., 2001. Orthostatic intolerance and motion sickness after parabolic flight. J. Appl. Physiol., 90, pp.67-82.

Schmäl, F., 2013. Neuronal mechanisms and the treatment of motion sickness. Pharmacology, 91(3-4), pp.229-241.

Simmons, R.G. et al., 2010. The efficacy of low-dose intranasal scopolamine for motion sickness. Aviat. Sp. Environ. Med., 81, pp.405-412.

Strewe, C. et al., 2012. Effects of parabolic flight and spaceflight on the endocannabinoid system in humans. Rev. Neurosci., 23, pp.673-680.

Suri, K.B., Crampton, G.H. \& Daunton, N.G., 1979. Motion sickness in cats: A symptom rating scale used in laboratory and flight tests. Aviat. Sp. Environ. Med., 50, pp.614-618.

Watson, D., Clark, L.A. \& Tellegen, A., 1988. Development and validation of brief measures of positive and negative affect: the PANAS scales. J. Pers. Soc. Psychol., 54, pp.1063-1070.

Weerts, A. et al., 2015. Restricted sedation and absence of cognitive impairments after administration of intranasal scopolamine. J. Appl. Physiol., in press.

Wood, C.D. et al., 1984. Side effects of antimotion sickness drugs. Aviat. Sp. Environ. Med., 55, pp.113-116. 
Table 1 - Pre and post PF scores on the questionnaires for the sopite case and the control group (Watson et al. 1988; Gianaros \& Muth 2001; Johns 1991).

\begin{tabular}{|c|c|c|c|}
\hline \multicolumn{2}{|l|}{ Measure } & \multirow[t]{2}{*}{ Sopite case $(n=1)$} & \multirow{2}{*}{$\begin{array}{c}\text { Group }(n=26) \\
\text { Lower and upper bound of the 95\% P.I. * }\end{array}$} \\
\hline MSAQ gastric (\%) & Pre & & \\
\hline \multirow{3}{*}{ MSAQ central (\%) } & Post & 83.33 & {$[0,66.91]$} \\
\hline & Pre & & \\
\hline & Post & 73.33 & {$[0,49.01]$} \\
\hline \multirow[t]{2}{*}{ MSAQ peripheral (\%) } & Pre & & \\
\hline & Post & 70.37 & {$[0,63.69]$} \\
\hline \multirow[t]{2}{*}{ MSAQ sopite-related (\%) } & Pre & & \\
\hline & Post & 100 & {$[0.79,32.65]$} \\
\hline \multirow[t]{2}{*}{ Overall MSAQ (\%) } & Pre & & \\
\hline & Post & 77.78 & {$[4.75,46.34]$} \\
\hline \multirow[t]{2}{*}{ ESS } & Pre & 10 & {$[0.25,13.35]$} \\
\hline & Post & 23 & {$[0,16.92]$} \\
\hline \multirow[t]{2}{*}{ PANAS positive } & Pre & 29 & {$[25.20,44.48]$} \\
\hline & Post & 16 & {$[26.91,46.21]$} \\
\hline \multirow[t]{2}{*}{ PANAS negative } & Pre & 24 & {$[6.14,28.42]$} \\
\hline & Post & 33 & {$[7.39,15.89]$} \\
\hline
\end{tabular}

PF: parabolic flight; MSAQ: Motion Sickness Assessment Questionnaire; ESS: Epworth Sleepiness Scale; PANAS: Positive and Negative Affect Scale; P.I.: prediction interval. The MSAQ scores are expressed in \%. Values of the sopite case outside the $95 \%$ prediction interval are highlighted in bold. * negative values of the lower bound of the $95 \%$ P.I. were changed to 0 as negative values do not hold a physical meaning for questionnaire scores. 